\title{
Actin Polymerization Is Essential for Myelin Sheath Fragmentation during Wallerian Degeneration
}

\author{
Junyang Jung, ${ }^{1 \star}$ Wenting Cai, ${ }^{1 \star}$ Hyun Kyoung Lee, ${ }^{1 \star}$ Marta Pellegatta, ${ }^{2}$ Yoon Kyung Shin, ${ }^{1}$ So Young Jang, ${ }^{1}$ \\ Duk Joon Suh, ${ }^{1}$ Lawrence Wrabetz, ${ }^{2}$ M. Laura Feltri, ${ }^{2}$ and Hwan Tae Park ${ }^{1}$ \\ ${ }^{1}$ Department of Physiology, Mitochondria Hub Regulation Center, College of Medicine, Dong-A University, Busan 602-714, South Korea, and ${ }^{2}$ Division of \\ Genetics and Cell Biology, San Raffaele Scientific Institute, 20132 Milan, Italy
}

The mechanisms that trigger Wallerian degeneration (WD) of peripheral nerves after injury are not well understood. During the early period of WD, fragmentation of myelin into ovoid structures occurs near the Schmidt-Lantermann incisures (SLI), a noncompact region of the myelin sheath containing autotypical adherens junction. In this study, we found that new filamentous actin polymerization occurs in the SLI of mouse sciatic nerves after injury and that its inhibition prevented not only the degradation of E-cadherin in the SLI but also myelin ovoid formation. However, the inhibition of actin polymerization could not block Schwann cell dedifferentiation. The activation of Rac GTPase was observed in the distal stump of the injured nerves, and a specific Rac inhibitor, a dominant-negative Rac, and Rac1-RNA interference blocked myelin ovoid formation. Together, these findings suggest that dynamic changes in actin in the SLI are essential for initiation of demyelination after peripheral nerve injury.

\section{Introduction}

As peripheral nerves develop, Schwann cells extend their processes into axon bundles to sort out an axon destined to be myelinated. Then, Schwann cells obtain a one-to-one relationship with the axon and form myelin by spiraling their membrane around the axon (Mirsky and Jessen, 1999). These morphological changes of Schwann cells are likely to be mediated by the actin cytoskeleton, which plays a key role in cell shape changes in response to various stimuli. Indeed, recent studies have demonstrated essential roles of Rho GTPases, major signaling molecules for actin polymerization, in axonal sorting and myelination in developing peripheral nerves (Benninger et al., 2007; Nodari et al., 2007). After completion of myelination, actin filaments remain mainly in the mesaxon, Schmidt-Lantermann incisures (SLIs), and nodal regions, suggesting that the actin cytoskeleton might be important for the maintenance of the domain structure of myelin sheaths (Trapp et al., 1989).

Wallerian degeneration (WD) following nerve injury involves axonal degeneration and degradation of the myelin sheath. Demyelination in Schwann cells starts mechanically with fragmentation of the myelin sheath into small ovoid-like structures and molecularly with the downregulation of myelin gene expression (Ghabriel and Allt, 1979a,b; Jessen and Mirsky, 2008). Morphologically, the fragmentation of myelin, including its disruption,

\footnotetext{
Received Aug. 31, 2010; revised Nov. 24, 2010; accepted Dec. 7, 2010.

This research was supported by Mid-career Research Program and Science Research Center through National Research Foundation grants funded by the Ministry of Education, Science and Technology of Republic of Korea (2010 - 0000132 and 2010 - 0001945) and by grants from the National Institute of Neurological Disorders and Stroke to M.L.F. (R01-NS045630) and L.W. (R01-NS055256).

*J.J., W.C., and H.K.L. contributed equally to this work.

Correspondence should be addressed to Dr. Hwan Tae Park, 3-1, Dongdaesin-Dong, Seo-Gu, Busan, 602-714, South Korea.E-mail: phwantae@dau.ac.kr.

DOI:10.1523/JNEUROSCI.4537-10.2011

Copyright $\odot 2011$ the authors $\quad 0270-6474 / 11 / 312009-07 \$ 15.00 / 0$
}

first occurs near an SLI, such that the myelin ovoids are delimited by the previous locations of the SLI (Webster, 1965; Ghabriel et al., 1979b). Although authors have long observed this morphological change during demyelination, the molecular mechanisms of myelin fragmentation at SLI are largely unknown. Since cytoskeletal dynamics might be involved in the extensive morphological changes of Schwann cells during demyelination, we investigated the role of actin filaments in the disruption of SLI and subsequent myelin ovoid formation.

\section{Materials and Methods}

Materials. The materials for the experiment are described in supplemental Figure 1 (available at www.jneurosci.org as supplemental material).

In vitro Wallerian degeneration and teasing. Sciatic nerve explant cultures from adult male mice (C57BL/6) and nerve fiber teasing were performed as we have previously reported (Lee et al., 2009). For animal surgeries, all procedures were performed according to protocols approved by the Dong-A University Committee on Animal Research, which follows the guidelines for animal experiments established by the Korean Academy of Medical Science. A myelin ovoid in teased nerve fibers was defined as a fragment of myelin sheath that under a microscope appeared to be completely separated from adjacent myelin. The number of myelin ovoids was counted along a $200 \mu \mathrm{m}$ length of medium- to large-size, teased nerve fibers ( $\sim 100$ nerve fibers/group). The SEM was calculated from three independent experiments.

Immunofluorescent staining, Western blot analysis, reverse transcription PCR, electron microscopy, and Rac GTPase activity assay. Immunofluorescent staining, Western blot analysis, and reverse transcription PCR were performed as previously described (Lee et al., 2009). The PCR primers for P0, Krox20, and glyceraldehyde-3-phosphate dehydrogenase are designated in supplemental materials (available at www.jneurosci.org as supplemental material). The preparation for electron microscopy and glutathione $S$-transferase pull-down assays for Rho GTPases was performed as previously described (Nodari et al., 2007).

Filamentous actin staining. Filamentous actin (F-actin) in the teased nerve fibers was labeled with fluorescein phalloidin (1:1000) for $3 \mathrm{~h}$ at 
room temperature. To visualize the newly polymerized F-actin, preformed stable F-actin was labeled with a cell-permeable actinbinding protein, jasplakinolide, at $1 \sim 2 \mu \mathrm{M}$ for $3 \mathrm{~h}$ at $37^{\circ} \mathrm{C}$ in DMEM containing $10 \%$ fetal bovine serum (FBS). The explants were washed with PBS twice and then incubated in a drugfree DMEM containing 10\% FBS for the indicated time. The explants were fixed in $4 \%$ paraformaldehyde (PFA), teased, and the newly formed actin filaments were labeled with fluorescein phalloidin.

Electroporation into the sciatic nerves. Under anesthesia, DNA solutions $(1 \mu \mathrm{g} / \mu \mathrm{l})$ containing $0.1 \%$ fast green as a tracer were injected into the sciatic nerves by using a Hamilton syringe with a glass micropipette under a stereoscope. Following injection, tweezer-type electrodes (model 520, BTX) were placed to hold the sciatic nerves, and two square pulses $(10 \mathrm{~ms}$ and $180 \mathrm{~V})$ with $950 \mathrm{~ms}$ intervals were applied by using a pulse generator (ECM830, BTX). One day after transfections, the sciatic nerves were transected at a proximal side of injection sites and the distal stumps were collected for the indicated times. After fixation in $4 \%$ PFA, the transfected sciatic nerves were identified and teased under a fluorescent stereomicroscope (MVX10, Olympus).

Racl conditional knock-out mice. The generation of Schwann cell-specific Rac1-null mice has been described (Nodari et al., 2007).

Application of cytochalasin $D$ in the lesion nerve in vivo. After sciatic nerve axotomy, the lesion site of the distal stump of the sciatic nerve was inserted into a PVC (polyvinylchloride) tube (10 $\mathrm{mm}$ length), and then the tube and lesion site were stabilized by filling the lumen with gelfoam presoaked with $200 \mu \mathrm{M}$ cytochalasin D (CD). CD was injected into the tube 2 consecutive days after surgery. Three days after axotomy, nerves were removed, fixed with $4 \%$ PFA, and processed for immunofluorescent staining.

\section{Results}

\section{Actin polymerization is required for myelin sheath} fragmentation in vitro

We have previously reported that in ex vivo culture the appearance of myelin ovoids was accompanied by progression of WD (Lee et al., 2009). To explore the signals involved in WD, we screened $\sim 30$ drugs that could possibly inhibit the appearance of myelin ovoids in ex vivo explant cultures (supplemental Fig. $1 \mathrm{~A}$, available at www.jneurosci.org as supplemental material). We found that two drugs that inhibit actin polymerization, CD (5 $\mu \mathrm{M})$ and latrunculin A (LTA) $(5 \mu \mathrm{M})$, significantly suppressed the formation of myelin ovoids after $3 \mathrm{~d}$ in vitro (DIV) (Fig. $1 A, B$ ). Immunostaining against myelin basic protein (MBP) revealed intact myelin sheaths in control (Fig. 1C) (0 DIV), whereas the staining was decreased and found in ovoids or clustered structures at 3 DIV. The intact myelin-like MBP staining was significantly preserved in the CD-treated nerves (Fig. $1 C$ ). In addition, semithin sections of nerve explants showed that the majority of myelin seemed to be maintained in the presence of CD or LTA (Fig. 1D). Finally, CD indeed delayed myelin degradation as demonstrated by Western blot against MBP (supplemental Fig. $1 B$, available at www.jneurosci.org as supplemental material).
B
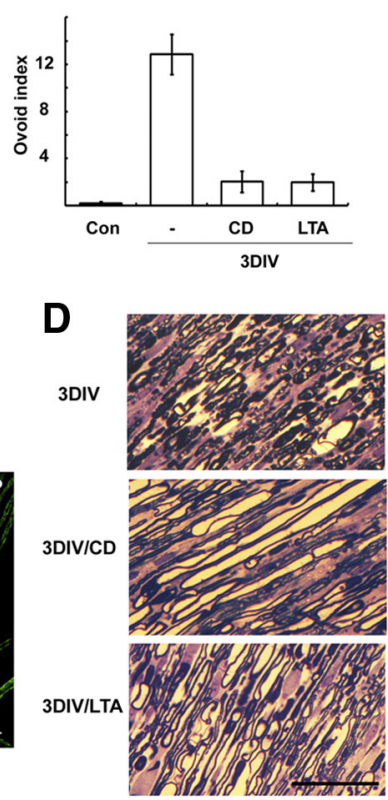

F

G
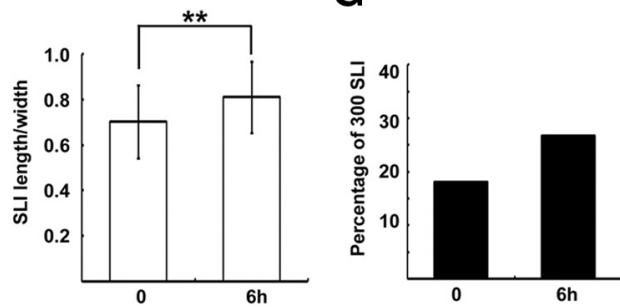

Figure 1. Actin polymerization is required for WD in vitro. $A$, Sciatic nerve explants were cultured for $3 \mathrm{~d}(3 \mathrm{DIV})$ in the absence of presence of $C D(5 \mu \mathrm{m})$, and then the explants were fixed, and teased nerve fibers were prepared. $\boldsymbol{B}$, Quantitative result of the (L) of F-actin-stained SLI were measured, and the index indicates length/width. ${ }^{* *} p<0.01$, Student's $t$ test. Erro bar indicates SD of 300 SLIs from three different experiments. G, The percentage of SLIs from the control (Con) and $6 \mathrm{~h}$ culture groups with the length/width indices $>1(N=300$ SLIs per group).

These findings suggest that actin polymerization is required for myelin fragmentation and degeneration. We thus examined actin dynamics in myelin-forming Schwann cells during WD after axotomy by staining teased nerve fibers with fluorescent phalloidin. In accordance with previous reports (Trapp et al., 1989; Fannon et al., 1995), we observed prominent F-actin staining in the SLI of uninjured control nerves, as identified by double staining with E-cadherin (supplemental Fig. 1C, available at www.jneurosci.org as supplemental material). The outer and inner perimeters of myelinated fibers were also mildly stained, whereas compact myelin was devoid of F-actin staining (Fig. $1 E$ ). At $6 \mathrm{~h}$ and $1 \mathrm{~d}$ after injury, the intensity of F-actin staining at the SLI increased and its shape appeared to be more funnel-shaped than that of the controls (Fig. 1E). We analyzed the shape of F-actin-labeled SLI by determining the length/width index of the SLI (300 SLIs per group) (Fig. $1 F$ ). Although the difference in the index between the control and $6 \mathrm{~h}$ experimental groups was not large ( $\sim 16 \%$ increase), it was statistically significant $(p<0.01, t$ test). We also determined the percentage of SLIs in which the length/ width index was more than one. Of 300 SLIs, 26.7 and 18\%, respectively, had indices $>1$ in the $6 \mathrm{~h}$ group and the uninjured control group (Fig. 1G). 
A
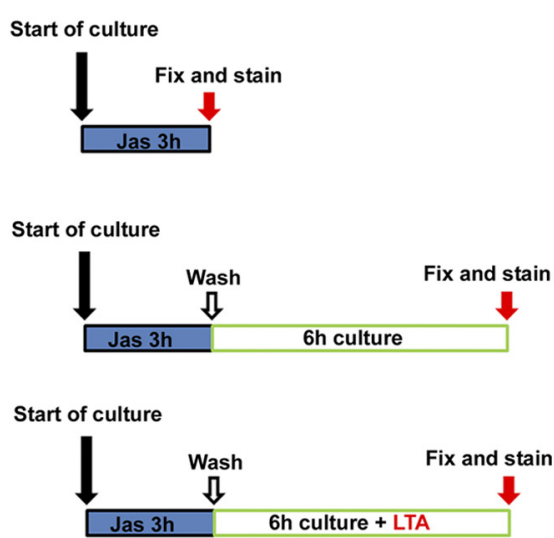

D

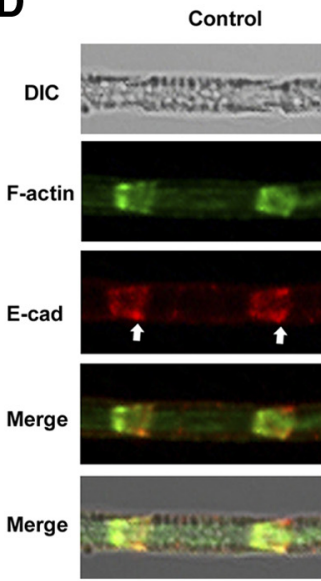

$6 \mathrm{~h}$ new- F-actin

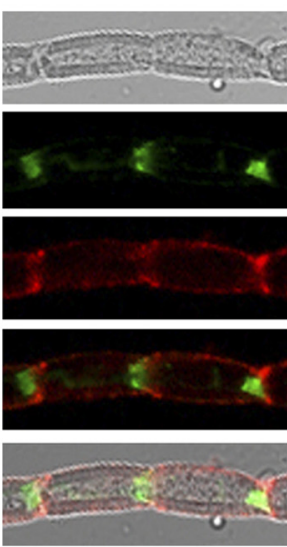

E

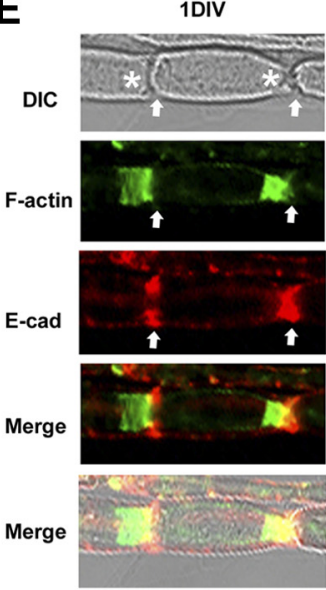

B

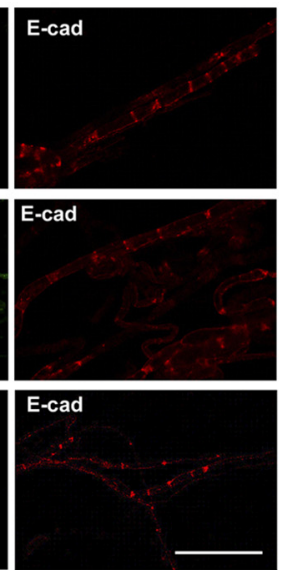

C

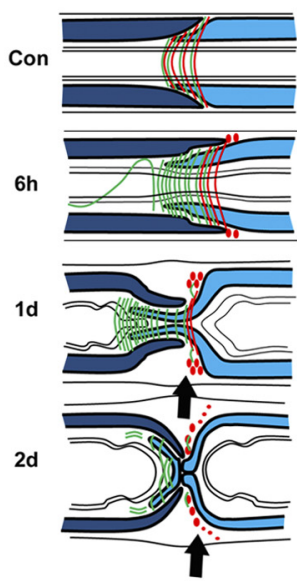

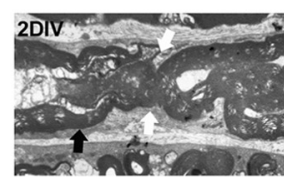
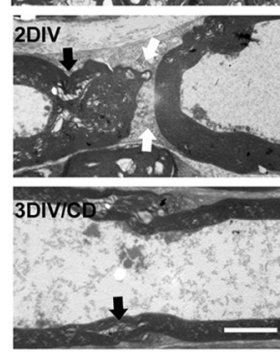

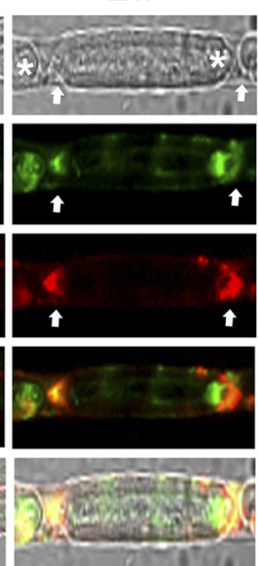

2DIVICD

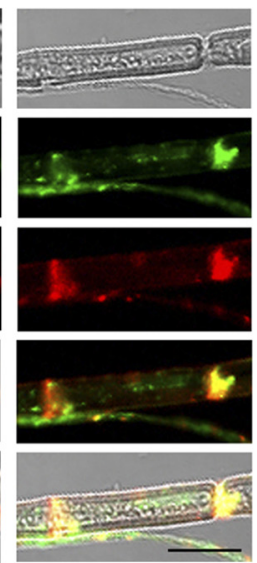

Figure 2. New F-actin formation during WD. A, Detection of newly polymerized F-actin. Sciatic nerve explants were cultured for $3 \mathrm{~h}$ in the presence of jasplakinolide (Jas) and double-stained with fluorescein phalloidin and an anti-E-cadherin antibody. A group of explants was washed following Jas treatment and cultured for another $6 \mathrm{~h}$ in the presence or absence of LTA. Scale bar, $100 \mu \mathrm{m}$. $\boldsymbol{B}$, Modeling of myelin ovoid formation. Red lines and red dots, E-cadherin; green lines, F-actin; black arrows, the rounding-off region. $\boldsymbol{C}$, Electron micrograph of cultured sciatic nerve explants. Black arrows indicate SLIs, whereas white arrows indicate the cytoplasmic areas around the rounding-off region. Scale bar, $2 \mu \mathrm{m}$. D, E, Teased nerve fibers were double-stained with fluorescein phalloidin and an anti-E-cadherin antibody. White arrows in the left panels are the wide region of SLls. Note the newly formed F-actin at $6 \mathrm{~h}$ in culture is found only in the inner narrow part of the $\mathrm{SLI}$ in the right panel of $\boldsymbol{D}$. At 1 and $2 \mathrm{~d}(\boldsymbol{E})$, the majority of F-actin was found at the end of developing myelin ovoids (asterisks), whereas E-cadherin was observed in the cytoplasm near the "rounding-off" region (arrows). Scale bar, $20 \mu \mathrm{m}$.

New F-actin formation in the sciatic nerve during the early period of WD

To selectively label newly polymerized actin during WD, we used jasplakinolide, a membrane-permeable drug that competes with phalloidin for F-actin binding (Dai et al., 2000). When jasplakinolide $(1 \mu \mathrm{M})$ was added for $3 \mathrm{~h}$ to sciatic nerve explants, phalloidin no longer labeled F-actin (Fig. $2 \mathrm{~A}$ ). We next allowed the explants to recover in jasplakinolide-free medium for various length of time, and then labeled new F-actin polymerization with fluorescent phalloidin. New F-actin was found in the SLI and on the inner/outer perimeters of Schwann cells within $3 \mathrm{~h}$ of washing out jasplakinolide, and the staining was increased further at $6 \mathrm{~h}$ (Fig. $2 A$; supplemental Fig. $2 A$, available at www.jneurosci.org as supplemental material). However, the new F-actin was not found in compact myelin and axoplasm (supplemental Fig. $2 A$, available at www.jneurosci.org as supplemental material). When the explants were incubated for $6 \mathrm{~h}$ in the presence of LTA after wash, no F-actin staining was detected, confirming that fluorescein phalloidin in this assay indeed recognized newly polymerized F-actin.

Because developing myelin ovoids are delimited by the SLI, SLI has long been suggested to be the cleavage site of the myelin sheath (Webster, 1965; Ghabriel et al., 1979b). Studies have revealed that myelin sheath cleavage occurs adjacent to incisures rather than within the SLI itself. In other words, ovoid formation occurs by "rounding off" of the peri-incisural myelin at the outer portion of the funnel-shaped SLI (Fig. $2 \mathrm{~B}$ ), and the inner narrow part of the SLI remains on the opposite side of the curvature of ovoid even after completion of ovoid formation. We also observed this asymmetric cleavage in ex vivo WD using electron microscopy (Fig. 2C).

Since the majority of newly polymerized F-actin was found around the SLI, we examined the spatial profile of F-actin changes in the SLI in detail. In control nerves, funnel-shaped SLIs were noted in differential interference contrast (DIC) microscopy, and F-actin seemed to fill the whole length of oblique bilateral bands of incisural cytoplasm with E-cadherin (Fig. 2D). New F-actin staining at $6 \mathrm{~h}$ showed that newly formed F-actin was found at the narrow part of the funnelshaped SLI (Fig. $2 B, D$ ). In contrast, E-cadherin staining was observed mainly in the outer part of the SLI. At 1 DIV, myelinated fibers showed incomplete myelin segmentation. Similar to the finding of F-actin staining at $6 \mathrm{~h}$, F-actin was 
A
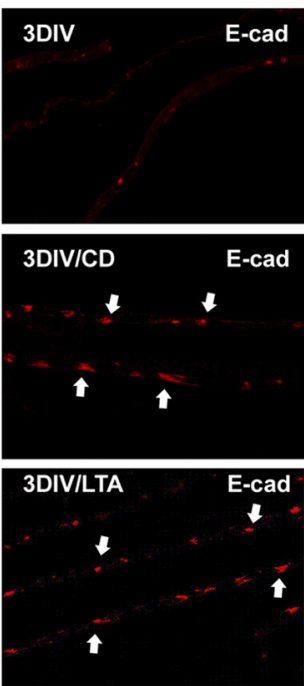

D
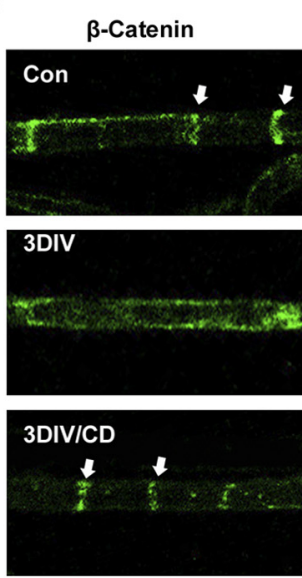
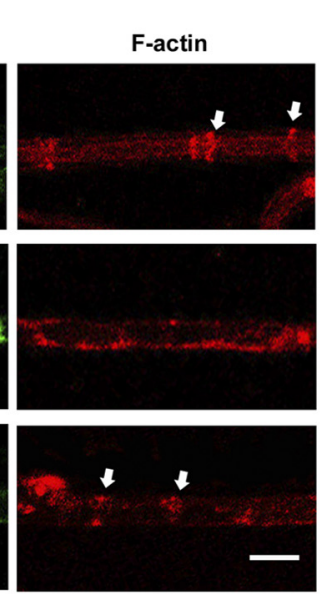
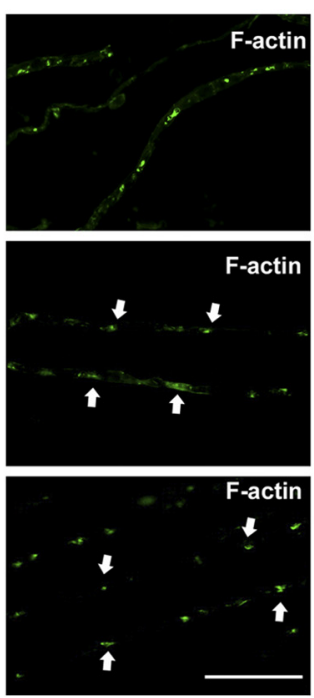

E
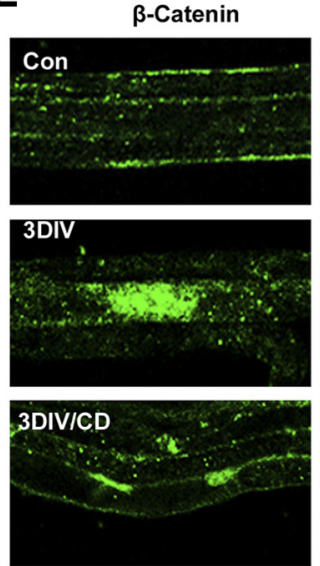

B

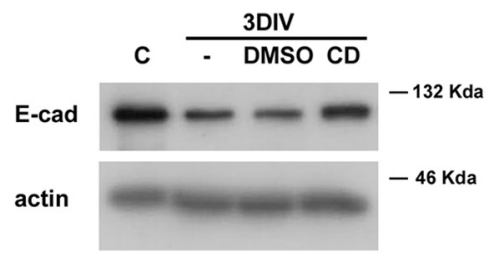

C

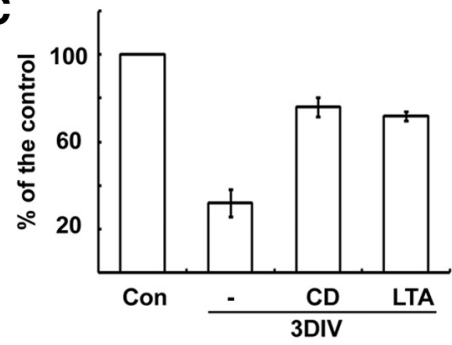

Figure 3. Actin polymerization-dependent regulation of E-cadherin and $\beta$-catenin in the SLI during WD. $\boldsymbol{A}$, Teased nerve fibers were immunolabeled with an antibody against $\mathrm{E}$-cadherin and fluorescein phalloidin. Arrows indicate the SLI where E-cadherin and F-actin are colocalized. Scale bar, $100 \mu \mathrm{m}$. $\boldsymbol{B}$, Protein extracts (10 $\mu \mathrm{g}$ ) from sciatic nerve explants were analyzed by Western blotting. Dimethylsulfoxide (DMSO) was the vehicle for drugs. $\boldsymbol{C}$, Quantitative analysis of Western blotting for E-cadherin expression from three independent experiments. $\boldsymbol{D}, \boldsymbol{E}$, Immunofluorescent staining against $\beta$-catenin in the teased nerve fibers from in vitro cultures. Arrows in $\boldsymbol{D}$ indicate SLI, whereas asterisks in $\boldsymbol{E}$ indicate the nucleus. Scale bar, $20 \mu \mathrm{m}$. $\boldsymbol{F}$, Quantitative analysis of the nuclear immunofluorescent staining against $\beta$-catenin from three independent experiments. The percentage of $\beta$-catenin-reactive nuclei from DAPI (diamidino-2-phenylindole dihydrochloride)positive nuclei ( 100 nuclei from each experiment) in the teased fibers was calculated. ( and Con, Control; E-cad, E-cadherin.

principally observed in the narrow part of the SLI, whereas E-cadherin seemed to be localized to the peri-incisural widened cytoplasmic region, which was near the "rounding-off region" (Fig. $2 B, E$ ). At $2 \mathrm{~d}$ after culture, ovoid formation was well established and two adjacent ovoids were completely separated. Thus, it was difficult to distinguish the rounding-off region and the narrow actin-rich region in the adjacent ovoid under DIC microscopy. However, the staining revealed that F-actin was still found within myelin ovoids and localized to one end of the ovoid, representing the remaining SLI within the ovoids (Fig. 2E, asterisks). In contrast, E-cadherin was localized to the narrow cytoplasmic region between two adjacent ovoids (Fig. $2 C, E$, white arrows). We postulate that actin polymerization might be required for the above processes of myelin fragmentation. Indeed, the inhibition of actin polymerization with CD resulted in the preservation of the general structures of the SLI, as demonstrated by ultrastructural analysis and immunofluorescent staining at 3 DIV (Fig. 2C,E).
Actin polymerization-dependent regulation of E-cadherin and $\beta$-catenin in the SLI during WD

The junctional proteins in the SLI, such as E-cadherin, seem to be essential for the maintenance of the structure of the SLI (Tricaud et al., 2005). We found that the staining intensity of E-cadherin was reduced with time in ex vivo cultures (Fig. $3 A$ ). This finding is consistent with the downregulation of E-cadherin levels in the sciatic nerve after injury (Hasegawa et al., 1996). In the present study, Western blot analysis revealed that the degradation of E-cadherin in explant cultures was inhibited by blocking actin polymerization (Fig. $3 B, C$ ). Furthermore, the colocalization of F-actin and E-cadherin in the SLI was preserved by actin polymerization inhibitors (Figs. 2E, 3A). We next examined a possible change in the expression of catenins in the sciatic nerves following injury. The protein levels of p120 catenin and $\beta$-catenin were not significantly altered, as demonstrated by Western blot analysis (data not shown). Interestingly, we observed a downregulation of $\beta$-catenin staining in the SLI with a 
concomitant nuclear translocation of $\beta$-catenin in the sciatic nerves after injury in vivo and during in ex vivo cultures with an actin polymerization-dependent manner (Fig. 3D-F).

Axonal degeneration and myelin degradation might be required for the initiation of Schwann cell dedifferentiation after injury (Jessen and Mirsky, 2008). Thus, we hypothesized that dedifferentiation of Schwann cells could be suppressed by actinperturbing agents. As shown in supplemental Figure 2 (available at www.jneurosci.org as supplemental material), neither ERK (extracellular signal-regulated kinase) activation nor induction of c-Jun expression (two early markers of Schwann cell dedifferentiation) (Harrisingh et al., 2004; Parkinson et al., 2008) in sciatic nerve explants was blocked by the inhibition of actin polymerization. Furthermore, the downregulation of myelin protein $\mathrm{P} 0$ and Krox 20 gene expression at 3 DIV was not prevented by the inhibition of actin polymerization. Thus, it seems that the role of actin polymerization in WD is specifically related to the disassembly of the SLI, rather than broad involvement in the dedifferentiation of Schwann cells.

\section{Rac GTPase-dependent actin polymerization is implicated in myelin ovoid formation in vivo}

Actin polymerization in Schwann cells after nerve injury may involve GTPases of the Rho subfamily. It was previously reported that Rac and Cdc42 are the major RhoGTPases expressed in adult peripheral nerves (Terashima et al., 2001). We thus examined the changes in Rac and Cdc42 activity in sciatic nerves after injury using a pull-down assay (Fig. $4 A$ ). Racl activity increased within $1 \mathrm{~h}$ following injury and remained elevated until $3 \mathrm{~h}$, whereas Cdc42 activity was not increased by nerve injury. We further determined Racl localization in the SLI following injury. Interestingly, the localization of green fluorescent protein (GFP)tagged Racl and endogenous Rac1 in the SLI was increased and obviously traversed the whole width of the myelin sheath in the SLI within $3 \mathrm{~h}$ of the injury (Fig. $4 B$; supplemental Fig. $3 A$, available at www.jneurosci.org as supplemental material), suggesting that Racl may be further recruited into SLI after injury. It is plausible that the widening of SLI following nerve injury (Ghabriel and Allt, 1979a) may be related to the increased localization of Rac1 to the SLI. To determine the role of Rac1 in myelin ovoid formation in vitro, we examined the effect of a specific Rac inhibitor, NSC23766 (100 $\mu \mathrm{M}$, Nodari et al., 2007). NSC23766 significantly inhibited new F-actin formation in SLI (Fig. 4C). Furthermore, the Rac inhibitor mimicked the preventive effect of $\mathrm{CD}$ on the myelin ovoid formation (Fig. $4 D$ ). In contrast, the Rho kinase inhibitor Y27632 had no significant effect. It was previously reported that neuregulin-ErbB2 signaling activated Rac in Schwann cells and played a role in myelin fragmentation (Guertin et al., 2005; Yamauchi et al., 2008). We found that a specific ErbB2 inhibitor, PKI166, significantly blocked not only the activation of Racl but also myelin ovoid formation in explant cultures (Fig. $4 E$; supplemental Fig. $1 A$, available at www.jneurosci. org as supplemental material), suggesting that ErbB2 signaling may be an upstream regulator of Rac1 activation in WD.

We next examined the effect of dominant-negative Rac1 (N17; Rac1DN) in myelin ovoid formation in vivo. After sciatic nerves were transfected with Rac1DN-GFP or GFP vector using electroporation, the axotomy-induced myelin ovoid formation was analyzed in the transfected myelinated fibers. We found that the transfection of Rac1DN significantly reduced the formation of myelin ovoids compared to GFP-transfected myelinated fibers in vivo (Fig. $4 F, G$ ). In addition, electroporation of Rac1-RNA interference (RNAi) significantly reduced the development of myelin ovoids after injury when compared with scrambled RNA (Fig. 4G). However, the transfection of constitutive active Rac1 (V12; Rac1CA) did not induce the myelin ovoid formation in uninjured nerves (Fig. 4F). We think that myelin fragmentation should accompany axonal degeneration within the myelin sheath. Thus, a possible alteration of myelin sheath structure by the overexpression of Rac1CA in Schwann cells could be insufficient for myelin fragmentation because of intact axonal cytoskeletal structures within the myelin sheath. The sum of these findings strongly suggests that Racl is an important upstream regulating molecule, but is not sufficient to induce myelin fragmentation in the SLI after injury. Schwann cell-specific Rac1-null mice show severely delayed development of the myelin sheath (Benninger et al., 2007; Nodari et al., 2007). We observed that SLIs were still found in hypomyelinated fibers of the conditional Rac1 knock-out mice even though their formation was rudimentary, as demonstrated by F-actin and E-cadherin staining (supplemental Fig. 3, available at www.jneurosci.org as supplemental material). Three days after axotomy, the degradation of E-cadherin and the appearance of ovoid-like structures were still observed in the Racl conditional null mice, suggesting that compensatory actions of other RhoGTPases or actinregulating molecules participate in myelin ovoid formation in this mutant. To confirm that actin polymerization is essential for myelin fragmentation in vivo, we applied CD to the distal stump of axotomized nerves for $3 \mathrm{~d}$ and performed immunofluorescent staining of teased nerve fibers (Fig. $4 H, I$ ). The degradation of E-cadherin and the formation of myelin ovoids were significantly delayed by the local application of CD to the lesion sites.

\section{Discussion}

How might actin polymerization in SLIs alter the structure of incisures, thereby leading to myelin fragmentation during WD? We suspect that actin polymerization-dependent E-cadherin destruction may be an underlying mechanism. The SLI contains atypical adherens junction (AJ) because membrane-to-membrane contact is autotypical (both membranes at a given junction from the same Schwann cells) (Fannon et al., 1995). However, the molecular nature of an SLI seems to be very similar to that of the typical AJ in epithelial cells. For example, cadherin/catenin complexes are abundantly expressed in SLI, and F-actin seems to provide a cytoskeletal framework for the maintenance of SLI as AJ (Fannon et al., 1995; Tricaud et al., 2005; Mège et al., 2006). Destruction of E-cadherin is a prerequisite for epithelial cells to lose their reciprocal contacts in epithelio-mesenchymal transition (Fischer and Quinlan, 1998; Braga et al., 2000). In addition, actin polymerization is essential for destruction of E-cadherin and subsequent disassembly of AJ in epithelial cells (Ivanov et al., 2004). Similar to these findings, it was recently reported that the ablation of E-cadherin signaling disrupted SLI (Tricaud et al., 2005). We found that E-cadherin was gradually removed from the SLI and accumulated in the Schwann cell cytoplasm near the rounding-off regions in an actin polymerization-dependent manner. Thus, it is possible that new actin polymerization in SLI leads to disassembly of the junctional complex-like structure by removing E-cadherin from SLI, thereby leading to changes in the membrane dynamics of the adjacent myelin sheath, and this disassembly may render the myelin sheath around SLI more susceptible to fragmentation. Interestingly, we detected nuclear translocation of $\beta$-catenin in the injured nerve. This process seems to be reminiscent of the epithelio-mesenchymal transition in epithelial cells (Willert and Jones, 2006), and further studies 


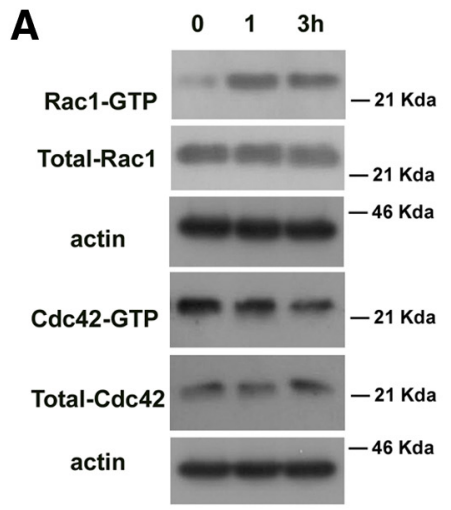

C
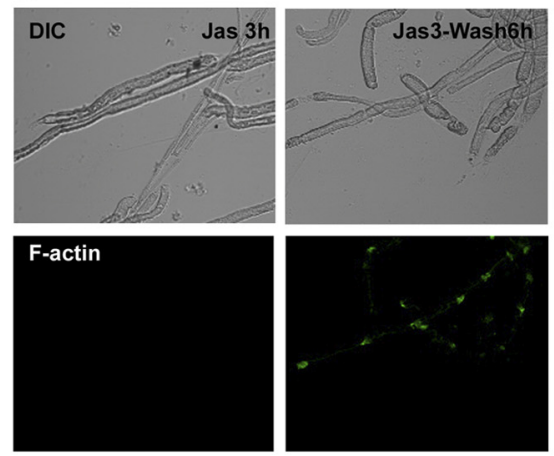

F
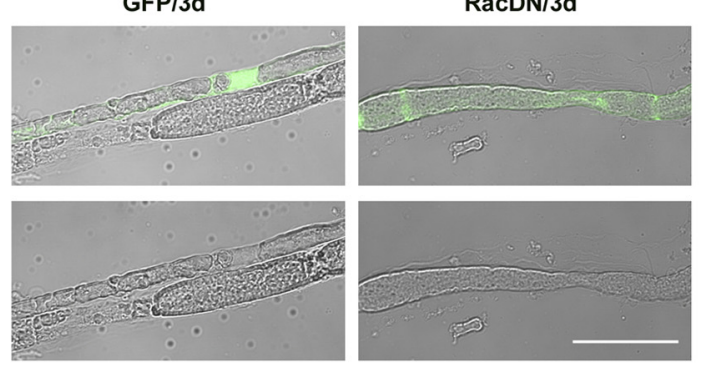

H
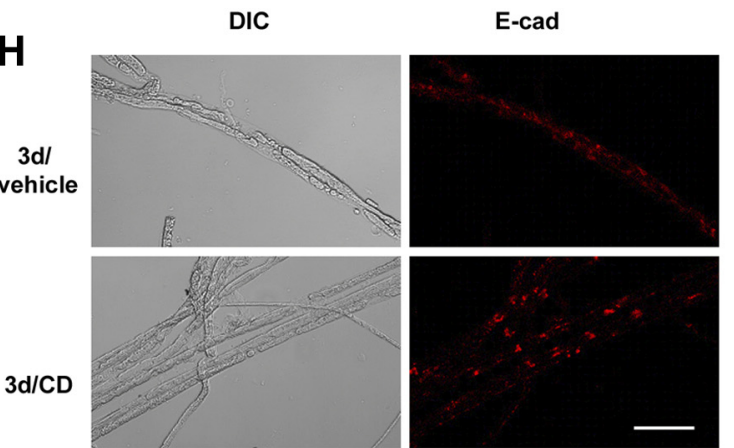

B
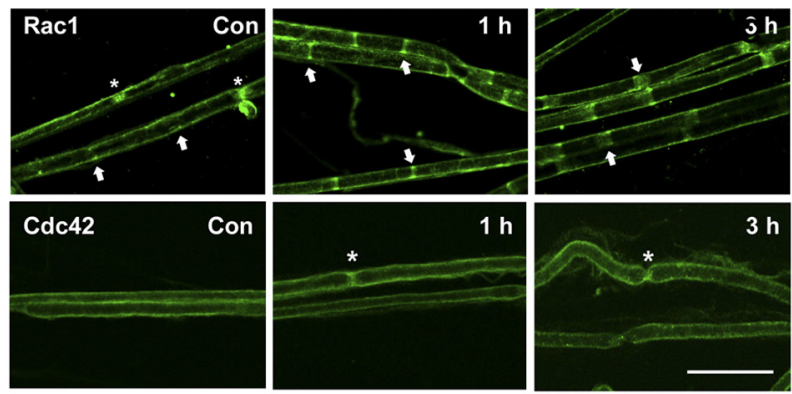

D

E
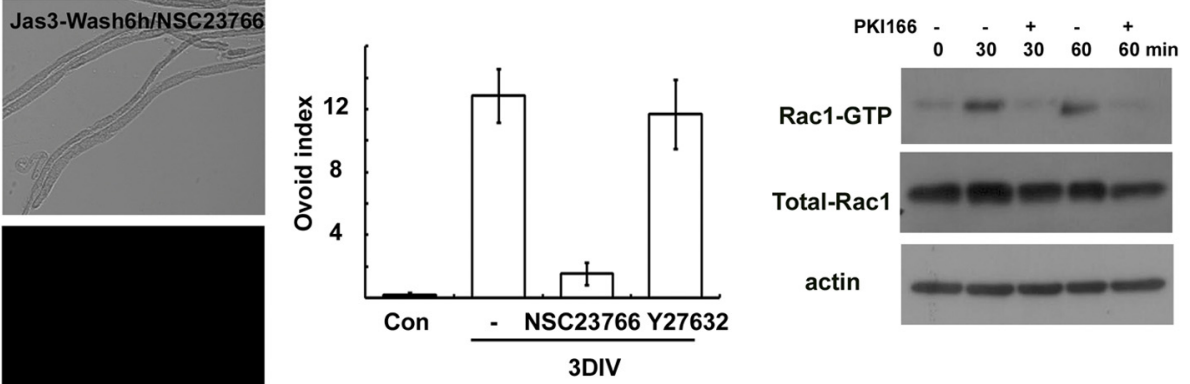
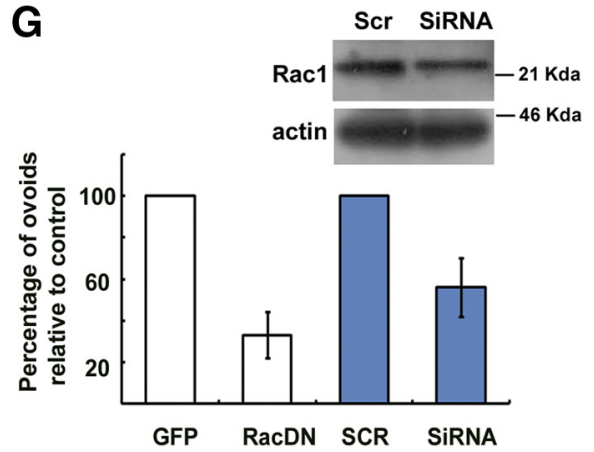

I

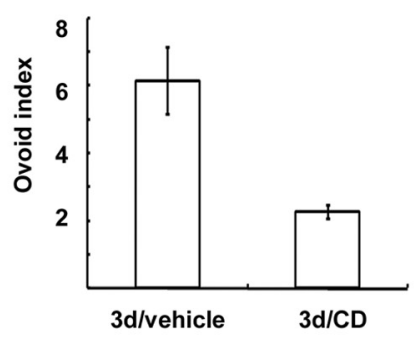

Figure 4. Role of Rac GTPase in myelin ovoid formation in vivo. $A$, Rac and Cdc42 activity assay. Levels of active Rac1 (Rac1-GTP) and Cdc42 (Cdc42-GTP) were measured using a pull-down assay. B, Immunofluorescent localization of Rac1 and Cdc42 in the rat sciatic nerves following nerve injury. Arrows indicate SLIs, whereas asterisks indicate Ranvier nodes. $\boldsymbol{C}$, New F-actin formation assay. Explants were treated with jasplakinolide for $3 \mathrm{~h}$ in vitro, and then the explants were further incubated for $6 \mathrm{~h}$ in the presence or absence of NSC23766. D, Myelin ovoid index showing the effect of NSC23766 and Y27632 (a Rho kinase inhibitor). $E$, Explants cultured for the indicated time in the presence of PKI 166 were assayed for Rac activity. $\boldsymbol{F}, \mathbf{G}$, Transfection of a dominant-negative Rac1-GFP (RacDN) or siRNA for Rac1 (G) inhibited formation of myelin ovoids. GFP indicates a GFP-vector control. G, Quantitative result showing the relative number of myelin ovoids to GFP or scrambled RNA (SCR) transfected controls. Western blot shows the effect of Rac1-RNAi on Rac1 expression in the transfected sciatic nerves. $\boldsymbol{H}$, After sciatic nerve axotomy, the lesioned nerve was treated with $C D$ or vehicle, and nerves were processed for immunofluorescent staining against E-cadherin. $\boldsymbol{I}$, Myelin ovoid index from lesioned nerve in vivo. Scale bar, $50 \mu \mathrm{m}$. 
will be required for elucidation of the role of $\beta$-catenin signaling in WD.

On the other hand, a role of axonal actin polymerization in myelin fragmentation can be excluded because of the following: (1) pre-existing F-actin and new F-actin in axons were minimal (Figs. 1E, 2D); (2) transfections of Rac-DN specifically into Schwann cells (supplemental Fig. $3 A$, available at www.jneurosci. org as supplemental material, shows the absence of GFP in axons) demonstrated a similar effect to that of actin polymerization inhibitors on nerve segments; and (3) an electron microscope analysis of cultured sciatic nerve segments revealed a typical degradation of cytoskeletal ultrastructures in the axoplasm following CD or LTA treatment (supplemental Fig. $4 A$, available at www. jneurosci.org as supplemental material), suggesting that axonal degeneration occurs normally in the presence of actin-perturbing agents. Thus, actin polymerization in Schwann cells is likely to be critical for myelin sheath fragmentation.

The suppression of myelin ovoid formation by blocking actin polymerization in Schwann cells could be the indirect result of an inhibition of proliferation, as demonstrated by supplemental Figure $4 B$ (available at www.jneurosci.org as supplemental material). However, a mitotic inhibitor, nocodazole, had no effect on myelin fragmentation (supplemental Fig. $1 \mathrm{~A}$, available at www.jneurosci.org as supplemental material), suggesting that Schwann cell proliferation might not be a prerequisite for myelin ovoid formation.

\section{References}

Benninger Y, Thurnherr T, Pereira JA, Krause S, Wu X, Chrostek-Grashoff A, Herzog D, Nave KA, Franklin RJ, Meijer D, Brakebusch C, Suter U, Relvas JB (2007) Essential and distinct roles for cdc42 and rac1 in the regulation of Schwann cell biology during peripheral nervous system development. J Cell Biol 177:1051-1061.

Braga VM, Betson M, Li X, Lamarche-Vane N (2000) Activation of the small GTPase Rac is sufficient to disrupt cadherin-dependent cell-cell adhesion in normal human keratinocytes. Mol Biol Cell 11:3703-3721.

Dai Z, Luo X, Xie H, Peng HB (2000) The actin-driven movement and formation of acetylcholine receptor clusters. J Cell Biol 150:1321-1334.

Fannon AM, Sherman DL, Ilyina-Gragerova G, Brophy PJ, Friedrich VL Jr, Colman DR (1995) Novel E-cadherin-mediated adhesion in peripheral nerve: Schwann cell architecture is stabilized by autotypic adherens junctions. J Cell Biol 129:189-202.

Fischer RS, Quinlan MP (1998) Identification of a novel mechanism of regulation of the adherens junction by E1A, Racl, and cortical actin filaments that contributes to tumor progression. Cell Growth Differ 9:905-918.

Ghabriel MN, Allt G (1979a) The role of Schmidt-Lanterman incisures in Wallerian degeneration. I. A quantitative teased fibre study. Acta Neuropathol 48:83-93.

Ghabriel MN, Allt G (1979b) The role of Schmidt-Lanterman incisures in
Wallerian degeneration. II. An electron microscopic study. Acta Neuropathol 48:95-103.

Guertin AD, Zhang DP, Mak KS, Alberta JA, Kim HA (2005) Microanatomy of axon/glial signaling during Wallerian degeneration. J Neurosci 25:3478-3487.

Harrisingh MC, Perez-Nadales E, Parkinson DB, Malcolm DS, Mudge AW, Lloyd AC (2004) The Ras/Raf/ERK signalling pathway drives Schwann cell dedifferentiation. EMBO J 23:3061-3071.

Hasegawa M, Seto A, Uchiyama N, Kida S, Yamashima T, Yamashita J (1996) Localization of E-cadherin in peripheral glia after nerve injury and repair. J Neuropathol Exp Neurol 55:424-434.

Ivanov AI, McCall IC, Parkos CA, Nusrat A (2004) Role for actin filament turnover and a myosin II motor in cytoskeleton-driven disassembly of the epithelial apical junctional complex. Mol Biol Cell 15:2639-2651.

Jessen KR, Mirsky R (2008) Negative regulation of myelination: relevance for development, injury, and demyelinating disease. Glia 56:1552-1565.

Lee HK, Shin YK, Jung J, Seo SY, Baek SY, Park HT (2009) Proteasome inhibition suppresses Schwann cell dedifferentiation in vitro and in vivo. Glia 57:1825-1834.

Mège RM, Gavard J, Lambert M. Regulation of cell-cell junctions by the cytoskeleton (2006) Curr Opin Cell Biol 18:541-548.

Mirsky R, Jessen KR (1999) The neurobiology of Schwann cells. Brain Pathol 9:293-311.

Nodari A, Zambroni D, Quattrini A, Court FA, D’Urso A, Recchia A, Tybulewicz VL, Wrabetz L, Feltri ML (2007) Betal integrin activates Racl in Schwann cells to generate radial lamellae during axonal sorting and myelination. J Cell Biol 177:1063-1075.

Parkinson DB, Bhaskaran A, Arthur-Farraj P, Noon LA, Woodhoo A, Lloyd AC, Feltri ML, Wrabetz L, Behrens A, Mirsky R, Jessen KR (2008) c-Jun is a negative regulator of myelination. J Cell Biol 181:625-637.

Terashima T, Yasuda H, Terada M, Kogawa S, Maeda K, Haneda M, Kashiwagi A, Kikkawa R (2001) Expression of Rho-family GTPases (Rac, cdc42, RhoA) and their association with p-21 activated kinase in adult rat peripheral nerve. J Neurochem 77:986-993.

Trapp BD, Andrews SB, Wong A, O'Connell M, Griffin JW (1989) Colocalization of the myelin-associated glycoprotein and the microfilament components, F-actin and spectrin, in Schwann cells of myelinated nerve fibres. J Neurocytol 18:47-60.

Tricaud N, Perrin-Tricaud C, Brusés JL, Rutishauser U (2005) Adherens junctions in myelinating Schwann cells stabilize Schmidt-Lanterman incisures via recruitment of p120 catenin to E-cadherin. J Neurosci 25:3259-3269.

Webster HF (1965) The relationship between Schmidt-Lantermann incisures and myelin segmentation during Wallerian degeneration. Ann N Y Acad Sci 122:29-38.

Willert K, Jones KA (2006) Wnt signaling: is the party in the nucleus? Genes Dev 20:1394-1404.

Yamauchi J, Miyamoto Y, Chan JR, Tanoue A (2008) ErbB2 directly activates the exchange factor Dock7 to promote Schwann cell migration. J Cell Biol 181:351-365. 